EDITORIAL of IA2030 and zero malaria

npj Vaccines (2020)5:109; https://doi.org/10.1038/

s41541-020-00259-3

This collection of malaria vaccine research and innovation papers highlights the intersection of efforts to: (1) achieve the panAfrican 1 and global goal ${ }^{2}$ of "Zero Malaria"; and (2) make the compelling case for immunization ${ }^{3}$ as a set of new tools for malaria control and elimination.

\section{PROBLEM AND OPPORTUNITY STATEMENT}

Despite a $\sim 30 \%$ reduction in clinical cases and $\sim 60 \%$ reduction in lives lost from Plasmodium ssp. infections over the past two decades, malaria continues to relentlessly sap the well-being of an estimated 228 million people worldwide (95\% confidence interval [CI]: 206-258 million) and result in the global demise of an estimated 405,000 persons annually. The effort, supported by a US\$ 2.7-3.2 billion annual investment, to "bend the curve" towards zero malaria by 2030 has stalled over the past five years ${ }^{4}$. This plateau is reminiscent of the decades-long effort to break through the $\sim 80 \%$ ceiling on reaching the goal of fully immunizing every child worldwide. Despite immunization of 116 million children annually, 20 million infants fail to receive a full course of essential vaccines and 13 million infants receive no vaccines whatsoever-these "zero dose" children are highlighted in the Immunization Agenda $2030^{3}$ (IA2030), recently adopted by the World Health Assembly ${ }^{5}$. The shared state of stalled progress towards equity and coverage of effective malaria interventions and essential vaccines has a common solution-new tools.

The early promise of innovative vector control and single-dose radical cure for malaria have yet to deliver the impact needed to achieve "zero malaria". That said, steadily over the last two decades, as reviewed by Duffy and Gorres, a pipeline of vaccine candidates against the most lethal human malaria, $P$. falciparum, and the most prevalent, $P$. vivax, has been assembled ${ }^{6}$. Novel vaccine platforms (e.g., $P$. berghei sporozoite-based human vaccine candidates $^{7}$ ), better defined correlates of protection in nonhuman primates ${ }^{8}$, and orthogonal learnings from other mosquitotransmitted pathogens (e.g., West Nile Virus) ${ }^{9}$ portend an even more robust pipeline of needed new tools, if adequate investments are made.

\section{P. FALCIPARUM VACCINE DEVELOPMENT STAGE-BY-STAGE AND STEP-BY-STEP}

Malaria vaccine targets are typically assigned to one of three sequential stages of the parasite's lifecycle: blood stage-through which the parasite causes all human pathology; sexual and sporogony stage-through which the parasite is transmitted from human host to female mosquito vector; and, pre-erythrocytic stage-through which the parasite infects humans during a subsequent bloodmeal by an infected mosquito (see Fig. 1, ref. ${ }^{6}$ ). Despite decades of efforts directed against targets throughout the lifecycle, only one vaccine candidate, the pre-erythrocytic circumsporozoite (CSP)-based RTS,S/ASO1 $1_{\mathrm{E}}$, has advanced through licensure and pilot implementation ${ }^{6}$. A myriad of biological and technical barriers have been encountered; however, step-by-step, these barriers have been chipped away. Advances include: new insights into the evolution of the immune response to the major pre-erythrocytic target, $\mathrm{CSP}^{10}$, and into the role of complementfixing antibodies in blood stage clinical immunity ${ }^{11}$; the ability to express and manufacture full length versions of blood stage targets, e.g., the highly promising, highly conserved reticulocytebinding protein homolog 5 (PfRH5) $^{12}$, as well as the 196-kDa merozoite surface protein 1 (the primary structure first described by Holder, et al. in $1985^{13}$ ), the latter of which demonstrated favorable safety and immunogenicity in a first-in human study ${ }^{14}$; and, the delivery of target malaria parasite antigens by measles vectors to overcome the barrier of waning immune responses, resulting in durable memory and protection, at least in a murine model ${ }^{15}$.

To be clear, significant challenges remain-for example, immune interference when concomitantly administering other vaccines, such as BCG for tuberculosis ${ }^{16}$, or administering multistage targets by multiple vaccine platforms, as observed with an adjuvanted virus-like particle, $\mathrm{RTS}, \mathrm{S} / \mathrm{ASO}_{\mathrm{B}}$, and viral-vectors expressing the multiple-epitope thrombospondin-related adhesion protein (ME-TRAP) ${ }^{17}$. That said, molecular approaches to identify and direct immune responses to specific promising epitopes $^{18}$ and use of novel particle-forming lipid-based adjuvants $^{19}$ provide paths forward for poorly immunogenic targets, including those designed to interrupt transmission from human to mosquito.

\section{MALARIA VACCINE USE CASES-PREGNANCY MALARIA MUST NOT BE LEFT BEHIND}

The longstanding mindset that vaccine evaluation during pregnancy should be delayed to post-licensure studies is obsolete. Even when pregnant women and their offspring do not have a higher risk of disease, there is an ethical rationale to evaluate at least vaccine safety pre-licensure ${ }^{20}$. Pregnancy malaria presents an even clearer case for prioritizing vaccine development as both mother and offspring have a well-described higher risk of disease ${ }^{6}$. More than two decades ago, antibodies to VAR2CSA, a member of the $P$. falciparum erythrocyte membrane protein 1 (PfEMP1) family, were associated with protection, identifying VAR2CSA as a promising vaccine target ${ }^{21}$. Placenta malaria vaccine (PMVs) research and innovation continues to progress ${ }^{6}$, including through the development of new animal models ${ }^{22}$ and down-selection of lead PVM candidates ${ }^{23}$.

\section{P. VIVAX - THE OTHER MALARIA}

Despite being the most prevalent human malaria parasite, investments in $P$. vivax vaccine development have been significantly smaller than that in the more lethal, albeit similarly morbid, falciparum cousin. Many $P$. falciparum vaccine targets have homologs in $P$. vivax ${ }^{6}$, so a fast follow-on vivax malaria vaccines based on safe, effective, affordable falciparum malaria vaccines have a reasonable, high likelihood of success. That said, several distinct differences in P. vivax biology, such as liver stage hypnozoites and rapid development of sexual stages directly from 
liver schizonts, require a $P$. vivax-specific vaccine research and innovation strategy. A critical differential feature of blood-stage vivax parasites is the use of Duffy antigen receptor for chemokines (DARCs) on human reticulocytes as a major invasion pathway. Recent insights into the structural basis of anti-P. vivax Duffy Binding Domain (PvDBP) immunity ${ }^{24,25}$ and early clinical results from a PvDBP vaccine candidate ${ }^{26}$ are encouraging; however, given the parasite's gene amplification mechanisms to evade antiPvDBP immunity ${ }^{27}$, developing a blood stage vivax malaria vaccine won't be trivial.

\section{RESEARCH AND INNOVATION AS A DRIVER FOR CREATING A COMPELLING VALUE PROPOSITION FOR MALARIA VACCINES}

The promising $P$. falciparum and $P$. vivax vaccine pipeline faces a significant resource shortfall as candidates head into late-stage development-this increasingly more apparent resource gap, or second "Valley of Death", is also faced by most, if not all, late stage vaccine candidates for pathogens afflicting primarily those living in low resource settings ${ }^{28}$. Hopefully, the recent global adoption of the Research and Innovation pillar of IA2030 and the growing African continent-led commitment to zero malaria will lead to the investments needed to generate the evidence that support the compelling value proposition required to build bridges for promising malaria vaccine candidates to become affordable, effective, sustainable new tools-part of the solution to regain the prior trajectory towards zero malaria.

Received: 24 October 2020; Accepted: 30 October 2020; Published online: 20 November 2020

\section{'PATH, 2201 Westlake Avenue, Suite 200, Seattle, WA 98121, USA. 凶email: dkaslow@path.org}

\section{REFERENCES}

1. RBM. Zero Malaria Starts with Me (RBM \& UNOPS, Geneva, 2018); https:// zeromalaria.africa/wp-content/uploads/2018/06/Agenda-setting-EN-1.pdf.

2. RBM. RBM Partnership to End Malaria Annual Report 2019 (RBM \& UNOPS, Geneva, 2020); https://endmalaria.org/sites/default/files/RBM\%20Annual\% 20Report\%202019.pdf.

3. WHO. Immunization Agenda 2030: A Global Strategy to Leave No One Behind (WHO, Geneva, 2020); http://www.who.int/immunization/immunization_agenda_ 2030/en/.

4. WHO. World malaria report 2019 (WHO, Geneva, 2019); https://www.who.int/ publications-detail-redirect/9789241565721.

5. WHO. Seventy-third World Health Assembly WHA73(9) Agenda item 11.3: Immunization Agenda 2030 (WHO, Geneva, 2020); https://apps.who.int/gb/ebwha/ pdf_files/WHA73/A73(9)-en.pdf.

6. Duffy, P. E. \& Patrick Gorres, J. Malaria vaccines since 2000: progress, priorities, products. NPJ Vaccines 5, 48 (2020).

7. Mendes, A. M. et al. A Plasmodium berghei sporozoite-based vaccination platform against human malaria. NPJ Vaccines 3, 33 (2018).

8. Douglas, A. D. et al. A defined mechanistic correlate of protection against Plasmodium falciparum malaria in non-human primates. Nat. Commun. 10, 1953 (2019).

9. Uraki, R., Hastings, A. K., Brackney, D. E., Armstrong, P. M. \& Fikrig, E. AgBR1 antibodies delay lethal Aedes aegypti-borne West Nile virus infection in mice. NPJ Vaccines 4, 23 (2019).

10. Murugan, R. et al. Evolution of protective human antibodies against Plasmodium falciparum circumsporozoite protein repeat motifs. Nat. Med. 26, 1135-1145 (2020).

11. Reiling, L. et al. Targets of complement-fixing antibodies in protective immunity against malaria in children. Nat. Commun. 10, 610 (2019).

12. Jin, J. et al. Production, quality control, stability, and potency of cGMP-produced Plasmodium falciparum $\mathrm{RH} 5.1$ protein vaccine expressed in Drosophila S2 cells. NPJ Vaccines 3, 32 (2018).
13. Holder, A. A. et al. Primary structure of the precursor to the three major surface antigens of Plasmodium falciparum merozoites. Nature 317, 270-273 (1985).

14. Blank, A. et al. Immunization with full-length Plasmodium falciparum merozoite surface protein 1 is safe and elicits functional cytophilic antibodies in a randomized first-in-human trial. NPJ Vaccines 5, 10 (2020).

15. Mura, M. et al. Recombinant measles vaccine expressing malaria antigens induces long-term memory and protection in mice. NPJ Vaccines 4, 12 (2019).

16. Walk, J. et al. Outcomes of controlled human malaria infection after BCG vaccination. Nat. Commun. 10, 874 (2019).

17. Rampling, $T$. et al. Safety and efficacy of novel malaria vaccine regimens of RTS,S/ AS01B alone, or with concomitant ChAd63-MVA-vectored vaccines expressing ME-TRAP. NPJ Vaccines 3, 49 (2018).

18. Canepa, G. E. et al. Antibody targeting of a specific region of Pfs47 blocks Plasmodium falciparum malaria transmission. NPJ Vaccines 3, 26 (2018).

19. Huang, W.-C. et al. Antibody response of a particle-inducing, liposome vaccine adjuvant admixed with a Pfs 230 fragment. NPJ Vaccines 5, 23 (2020).

20. Krubiner, C. B. et al. Pregnant women \& vaccines against emerging epidemic threats: ethics guidance for preparedness, research, and response. Vaccine S0264-410X, 30045-3 (2019).

21. Fried, M., Nosten, F., Brockman, A., Brabin, B. J. \& Duffy, P. E. Maternal antibodies block malaria. Nature 395, 851-852 (1998).

22. Doritchamou, J., Teo, A., Fried, M. \& Duffy, P. E. Malaria in pregnancy: the relevance of animal models for vaccine development. Lab Anim. 46, 388-398 (2017).

23. Chêne, A. et al. Down-selection of the VAR2CSA DBL1-2 expressed in E. coli as a lead antigen for placental malaria vaccine development. NPJ Vaccines 3, 28 (2018).

24. Rawlinson, T. A. et al. Structural basis for inhibition of Plasmodium vivax invasion by a broadly neutralizing vaccine-induced human antibody. Nat. Microbiol. 4, 1497-1507 (2019).

25. Urusova, D. et al. Structural basis for neutralization of Plasmodium vivax by naturally acquired human antibodies that target DBP. Nat. Microbiol. 4, 1486-1496 (2019)

26. Singh, K. et al. Malaria vaccine candidate based on Duffy-binding protein elicits strain transcending functional antibodies in a Phase I trial. NPJ Vaccines 3, 48 (2018).

27. Popovici, J. et al. Amplification of Duffy binding protein-encoding gene allows Plasmodium vivax to evade host anti-DBP humoral immunity. Nat. Commun. 11, 953 (2020).

28. Kaslow, D. C. et al. Vaccine candidates for poor nations are going to waste. Nature 564, 337-339 (2018).

\section{ACKNOWLEDGEMENTS}

This work was supported by the Bill \& Melinda Gates Foundation, Seattle, WA [OPP1180199]. The funder had no role in preparation of the manuscript or decision to publish.

\section{AUTHOR CONTRIBUTIONS}

DCK conceived, wrote, reviewed, approved submission of, and is accountable for this paper.

\section{COMPETING INTERESTS}

DCK is an employee of PATH (a not-for-profit organization), has no financial interest in any for-profit organization, and declares no competing interests. PATH is funded to innovate and partner in developing and implementing malaria vaccines and other interventions to control and eliminate malaria.

\section{ADDITIONAL INFORMATION}

Correspondence and requests for materials should be addressed to D.C.K.

Reprints and permission information is available at http://www.nature.com/ reprints

Publisher's note Springer Nature remains neutral with regard to jurisdictional claims in published maps and institutional affiliations. 
Open Access This article is licensed under a Creative Commons Ac Attribution 4.0 International License, which permits use, sharing,
adaptation, distribution and reproduction in any medium or format, as long as you give appropriate credit to the original author(s) and the source, provide a link to the Creative Commons license, and indicate if changes were made. The images or other third party material in this article are included in the article's Creative Commons license, unless indicated otherwise in a credit line to the material. If material is not included in the article's Creative Commons license and your intended use is not permitted by statutory regulation or exceeds the permitted use, you will need to obtain permission directly from the copyright holder. To view a copy of this license, visit http://creativecommons. org/licenses/by/4.0/.

(c) The Author(s) 2020 